\title{
The Standardized Nurse: Mission Impossible?
}

\author{
Rune Pedersen $^{1}$, Gunnar Ellingsen ${ }^{1}$, and Eric Monteiro ${ }^{2}$ \\ ${ }^{1}$ University of Troms $\varnothing$ Telemedicine Laboratory \\ rune.pedersen@uit.no gunnar.ellingsen@hn-ikt.no \\ ${ }^{2}$ Department of Computer and Information Science, \\ Norwegian University of Science and Technology \\ eric.monteiro@idi.ntnu.no
}

\begin{abstract}
Socio-technical approaches, with their over-emphasis on situated and contextual differences, find it difficult if not impossible to account for ICT-supported standardization of healthcare work. Empirically, not all efforts of standardization fail. How can that be theoretically conceptualized, even when key tenets of a situated perspective are maintained? We discuss an interpretative case study where standardization of nursing work-to an interesting degree-has been achieved. We analyze the process of co-construction of the standards (i.e., standards in practice). Standards are partly imposed from the top, and partly enacted through the active involvement and ingenuity of users.
\end{abstract}

Keywords: Standardization, nursing work, quality, dependency and efficiency.

\section{Introduction}

Perspectives on standardization of work tend to be polarized: either standardization is perceived as a typically top-down effort promoting "best practices" or, as often argued in socio-technical approaches to ICT in health care(Ellingsen et al. 2007; Timmermans and Berg 1997), standardization is considered futile, as health work is inherently situated and thus unique. Such a position is unsatisfactory for practitioners (they will find it hard to accept that any form of standardization is impossible) as well as inadequate from a research point of view. There is thus a sense in which standardized work in hospitals does not work in theory (i.e., the academic literature), but works in practice.

We wish to emphasize the importance of a theoretical perspective for the standardization of Information System embedded service work. The key questions this paper addresses are

(1) How can processes for standardization of healthcare work be described sociotechnically?

(2) How can one identify practical key implications of heterogeneous work practices to promote IT-based standardization?

Empirically, we draw on the broad efforts of standardization of healthcare work at Akershus University Hospital (AHUS), one of the largest hospitals in Norway. Since 
2005, the hospital has embarked on an ambitious effort aimed at a level of standardization of healthcare work unprecedented in Norway. Certainly, the boldest ambitions regarding standardization have not been achieved. However, significantly more standardization than the literature suggests has been achieved. Our empirical focus is on the standardization of nursing work, an IT-based system focusing on electronic patient records (EPR), and IT-based standardization of nursing plans and associated procedures for nursing. The standardization efforts also included efforts to associate standardized "pools" of patient beds and wards together with a generic pool of nurses.

\section{Conceptualizing Standardization in Healthcare}

Historically, IS research has a deeply embedded role in the ongoing transformation of modern organizations (Ellingsen et al. 2007). Standardization in information systems has a long history, from programming language, to communication protocols, to exchange formats (Schmidt and Werle 1998). There is an even stronger tradition of de facto standards for applications, operating systems, and file formats (Hanseth et al. 1996). However, an issue that has received considerably less attention in IS research is IS-based initiatives for the standardization of work and routines (Ellingsen et al. 2007; Timmermans and Berg 1997). Given the increasingly importance of the service sector in IS research, it is vital to extend the focus from standardization of artefacts and products to include standardized, IS-embedded service work-such as work processes in hospitals (Ellingsen et al. 2007). In addition, the maturing of technology alongside significant practical experiences substantiates current visions of a working e-infrastructure (Hepsø and Monteiro 2009).

In relation to the historical context, which entailed a technocratic top-down approach to standardization (Ure and Proctor 2009), the field has matured. We now see increasing consideration of a broader conceptualization in which standards form the backbone of socio-technical networks. Standardizing the work of nurses, physiccians, and other health workers has proven remarkably difficult to achieve, and is interwoven with efforts to improve efficiency and quality in health care (Bowker and Star 1999; Ellingsen et al. 2007; Timmermans and Berg 1997). Modern nursing is embedded in a highly politicized and institutionalized arena where governmental and managerial rules, regulations, and politics are negotiated against local concerns and priorities (Timmermans and Berg 2003). A fundamental characteristic of this work is its pragmatic, fluid character. Despite the obvious potential for improvements in efficiency, safety, and quality, standardization efforts seldom meet their objectives (IOM 2001). One reason for this is that health care in general and hospitals in particular are characterized by highly specialized-and thus unique-routines and procedures that differ across wards, areas, and geography. Like other complex work activities, health care is characterized by the constant emergence of contingencies that require ad hoc and pragmatic responses. Although much work follows routinized paths, the complexity of health care organizations and the never fully predictable nature of patients' reactions to interventions result in an ongoing stream of sudden events (Berg 1999). In her book on the process of developing from a novice to an expert, Patricia Benner (1984) has described how beginners and experts do things differently, and how a nurse's competence changes as her clinical career develops. This approach is described as 
underpinning the role of core personnel in the organization. Brenner's theory builds on the Dreyfus model, which illustrates the situational, experience-based premises that differentiate the levels of skilled performance. Learning from theory and from context-dependent judgement and skills are vital to this. In addition, health care work is typified by ongoing negotiations about the nature of the tasks and the relationships between those who execute the tasks (Button and Harper 1993). For example, Hughes (1988) has documented how experienced nurses often help inexperienced residents by suggesting the way to a diagnosis, or by hinting at the necessary treatment. Even though our interpretations do not compare nurses and physicians, the nurse versus nurse relation could be inherently situated.

However, the ever-increasing level of change in health care is problematic. Economic and qualitative requirements for treatment and care constantly challenge health care authorities to make increasingly strained efforts to maintain their budgets. Hence, the need to curb large and seemingly ever-increasing health care expenditures is an explicit feature of managerial agendas for the increased standardization of health care work (IOM 2001). In this regard, IS in general and nursing care plans (Berg 1999; Voutilainen et al. 2004) in particular are considered key means for standardizing nursing work to obtain increased efficiency as well as improved quality of care (Ellingsen et al. 2007).

While a great deal of the medical informatics literature, such as Kalra (2006), promotes a top-down approach to standardization, the STS and CSCW literature (Hanseth and Lundberg 2001; Pollock et al. 2007) promotes a more careful approach. Along these lines, Hanseth and Lundberg (2001) maintained that in organizations with particularly complex work practices, such as hospitals, only the users themselves would know the practice well enough to be able to design a system for the organization, resulting in a bottom-up approach to standardization.

Our aim is not to engage in a debate between the two extremes, but to position ourselves somewhere in the middle. The contexts of the use of EPR nursing plans are always individually different and characterized by highly idiosyncratic practices, whereas technologies are singular and monolithic; localization is the means by which the standard and the unique are brought together. One strand of research highlights the diversity of specific organizational contexts while others recognize that the apparently similar systems are being used across the same settings(Pollock et al. 2007). Several researchers believe that systems can merge across different practices equally; adjustments thus always need to be made. For instance, Pollock et al. (2007) have pointed out how large-scale systems can be successfully transferred across organizational boundaries. One example is a student administration system, the campus management model (GM) developed by the German software house SAP, which in the end was launched in the wider market as a "global university solution" (Pollock et al. 2007). Our position is to flag bottom-up approaches for health care institutions while at the same time recognizing the importance of top-down initiatives. Our intention is to develop an understanding of information systems embedded in the standardization of hospital work. From a co-constructive perspective on standardization, in which standardization and practice mutually shape and constitute each other, standardized work always involves "local universalities." Global standards both shape and are shaped by local work practice (Berg 1997). 


\section{Method}

The focus of our field work has been on the challenges constructed by the heterogeneity of hospital work, with the focus on IT, information systems standards such as the electronic patient record (EPR) based nursing plans, and interconnected work process standards such as generic pools for beds and personnel.

The importance of social issues related to computer-based information systems has been increasingly recognized over the last decade, and this has led IS researchers to adopt empirical approaches that focus particularly on human interpretation and meanings(Walsham 1995). In practice, the flow of health care work activities is often much less linear than it is in other arenas, as it has flexibly defined roles. Interpretive methods of research in IS are aimed at producing the information system, and the process whereby the information system influences and is influenced by the context. Interpretive research can help the IS researcher to understand human thought and action in social and organizational contexts (Klein and Myers 1999). Further, Orlikowski and Baroudi (1991) define interpretive research as follows: interpretive studies assume that people create and associate their own subjective and intersubjective meanings as they interact with the world around them. The interpretive researcher thus attempts to understand through accessing the meanings participants assign to them. Our study adheres to an interpretive research tradition of this nature. Interviews, observations, and document analysis have focussed on key actors, mostly nurses, physicians, and project managers, as well as architecture and artefacts. In general, qualitative research methods like interviews and observations are optimally suited to understand a phenomenon from the participants' point of view, and in particular the social and institutional context. Qualitative research techniques can provide deep insight, identify problems, and answer the why and the how questions that quantitative studies cannot answer (Ash et al. 2004).

Ethnography produces in- depth understanding of real-world social processes. Properly done, it provides detailed insight into the concepts and premises that underlie what people do-but that they are often unaware of (Forsythe 1999, p. 129)

\subsection{Data Collection}

We have employed four modes of data collection during a period from September 2009 to March 2010: observations, semi-structured interviews, document analysis, and analysis of central logs with general numbers on the use of nursing care plans.

In total, the first author conducted 170 hours of observations and 10 semistructured interviews averaging 80 minutes at the Cardiology department of AHUS in the period 2009-2010. The third author conducted six interviews at the Department of Pulmonary Medicine as a supplement to the standardization of work processes in the same period. The first author further conducted eight semi-structured interviews of personnel who had practical importance for the recommendation of a classification system for nursing, an initiative that in the future will secure a nationally embraced, consistent use of terminology in Norway. The interviews lasted between 60 and 140 minutes, with an average of 100 minutes. 
The length of the observations varied from between one and eight hours, and included tracing patient trajectories through the hospital to understanding the adoption and use of IT: based information carried out by nurses and physicians in different circumstances, such as between people, between people and technology, and between people and artefacts.

\subsection{Data Analysis}

The overall process of collecting data has been open-ended and iterative, with a gradually evolving focus on specific situations from work practice. Crucial to the evolving questioning has been interviews both among experienced nurses and novices. The analytical categories emerged from internal discussions and reading of field notes.

Essential to the case, and taking an interpretive position, is the subjective and intersubjective meanings taken by experienced nurses and novices. Handwritten field notes were transcribed shortly after the data was gathered. All transcriptions of the interviews were done immediately after the interviews themselves, as, according to Malterud (2003), early transcription is crucial in order to clarify uncertainties and the meaning of unclear sentences. The interviews were done using a tape recorder, and we posed only a few open-ended questions that were semi-structured and shaped according to how the interviews evolved.

Our findings have been discussed among fellow students, as well as discussions the first author with the second and third authors of this article, both of whom have a thorough understanding of and experience in working with IS studies and, specifically, with nursing plans, handovers, and classifications.

\subsection{Context of Study}

The research was conducted at the Akershus University Hospital (AHUS), which has about 4,700 employees and a total of 820 beds. Our study took place at the Division of Cardiology, specifically, the general Department of Cardiology, which consists of 28 beds.

The hospital is built on a model from Johns Hopkins Hospital in Baltimore, Maryland, globally acclaimed for its exceptional services and a program striving for standardization and efficiency in health care.

The reallocation of AHUS to new premises during 2008 brought additional initiatives to standardize nursing work, particularly to avoid bottlenecks caused by overly specialized services. One measure was to establish standardized beds and nurses for patients (i.e., the capacity to allocate patients to beds outside "their" wards and having nurses work in wards other than their "own"). To allocate patient beds, a new unit for patient logistics was established, which would book the beds for patients. As a rule, this unit would try to assign patients where they normally belonged. In cases of overbookings, the patient logistics unit prioritizes the serious incidents and moves less serious cases out of the "home" wards and into any of two generic bed wards. Here, nurses have special training in caring for patients with a wide variety of conditions, but no serious illnesses. As one nurse noted, "the biggest change was for the physicians, as they now also have to visit the generic bed wards. "Key mechanisms for 
volunteering nurses to circulate in other wards have been the incentives offered: salary and flexibility. Salaries are increased by an extra 5 percent for those who choose to circulate. Equally important, especially for family-oriented nurses, is that signing up for circulation gives nurses greater control over when they are to work.

From 2007, the responsibility for collecting blood specimens was, on the whole, decentralized to a departmental level and to the hospital's nurses. The potential effects of this were related to efficiency. Previously, technologists from the medical biochemistry laboratory collected blood specimens on fixed rounds three times a day, which resulted in delays and rigidity in the routines. In particular, blood specimens collected on an ad hoc basis or in emergencies had to be handled manually.

However, collecting blood specimens is a resource-demanding procedure that, one nurse complains, "has resulted in much more work for us, and is not time-saving." Still, the time from requesting a laboratory analysis until the result is available was greatly decreased throughout the first year. In addition, there were positive side effects. The nurses spend more time with the patients, and the patients no longer have to interact with so many different personnel.

\section{Standardization of Nursing Work}

\subsection{History of Standardized Nurse Work}

The EPR used at AHUS since 2005 includes a module for nursing (see Figure 1 for explanation). Along the lines of standardization, the nursing care plan, including the classification systems NIC and NANDA, was viewed as a means for making nursing work more effective and offering both quality assurance and future research capabilities. The classification systems are ICT-based standards integrated with the care plan. The diagnoses are represented by the international classification system of the North American Nursing Diagnosis Association (NANDA), consisting of 206 nursing diagnoses (NANDA 2007). The interventions are represented by the Nursing Intervention Classification (NIC) system, consisting of 486 interventions (Bulechek et al. 2008). Further, PPS is a decision support system for nursing, developed in collaboration with the authorities and distributed to educational institutions, municipalities, and hospitals nationwide.

The foundation of an independent nursing division was introduced in 2007 with its own spokesman on the hospital staff, established to sharpen the focus on nursing activities, such as utilization of resources, research, and development of expertise.

More recently, a national initiative stressed establishing a national incentive for recommending an all-encompassing classification system. The agenda of the initiative was first to get the National Directory of Health interested in nursing classifications and thereby included in their portfolio of classification systems, which first and foremost means financial support for further development. This process has detained the use of NIC and NANDA on a national level, and is a typical topic discussed and solved in the collaborating groups.

Several hospitals in Norway have used NIC and NANDA for a decade, and the vendor has estimated that, on a national level, they have more than 25,000 users of the nursing module. 


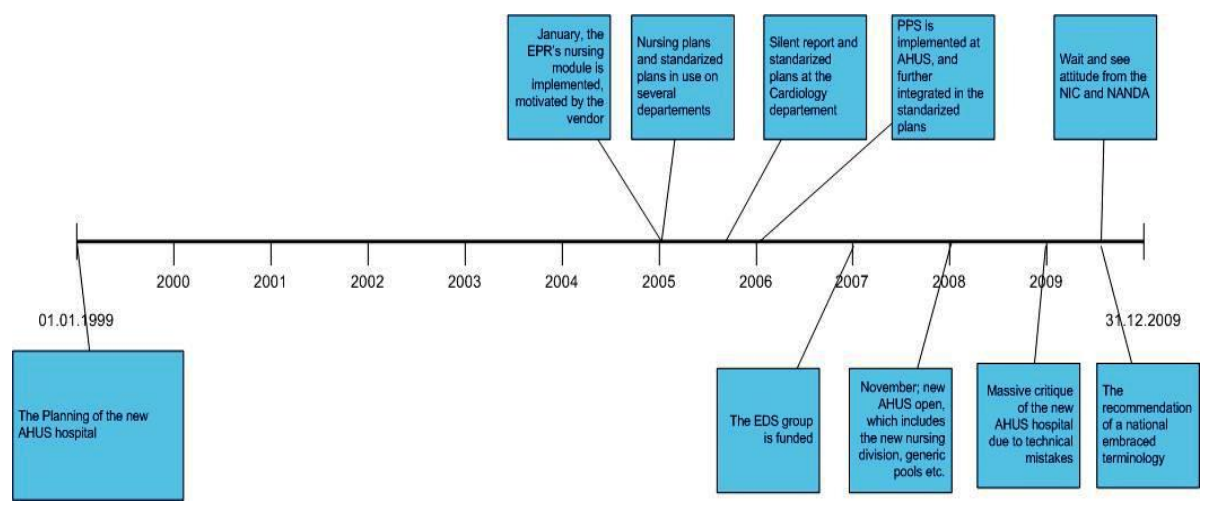

Fig. 1. Time line, from early planning of the new AHUS hospital and up to today

\subsection{The Care Plan}

Care plans are increasingly made to replace the use of free text in the documentation, foremost to establish a common, formalized language based on best practice. Freetext documentation is whatever information the nurses share about the patient in the EPR in addition to or without writing formalized care plans. However, the implementation of the EPR led to a systematic use of standardized care plans. Too much time was spent documenting unessential information (free-text) such as "eaten two slices of bread with jam and cheese" and "been for a walk."

The care plan has been organized such that each diagnosis, dimension, and action is firmly attached to the plan with a start and a stop date. When standardizing these plans, the nurse can easily choose several actions from a predefined list for the applicable diagnosis. By doing this, the nurse saves time, and at the same time the standardized sentences work as a quality indicator. Figure 2 illustrates the ordinary and the standardized plans respectively. Basically, the nursing plan is an overview of nurse-related diagnoses for a particular patient group combined with relevant interventions (NIC interventions, following the NANDA diagnosis).

\subsection{Standardized Plans and Silent Handover at the Cardiology Department}

Standardized plans and a silent handover were introduced as a "package" late in 2005 at the Cardiology department. Equal for all of the IT-based standards described in our case - the care plan, standardized plan, and PPS-is that they were adopted and used before the reallocation process started. The standardized plan represents a care-fully selected combination of NANDA diagnoses and NIC interventions for a given medical diagnosis or clinical speciality. A number of plans were made by and for each speciality, with 17 for cardiology, and 406 plans in total. Some plans are also generic, covering diagnosis and interventions for patients with composite diagnoses. The standardized plans have been added to a shared list (Figure 2 on the right). The list has further been synthesized into one continuous list with a common search engine where you search for specialities like medicine (MED) and surgery (KIR), and an applicable diagnosis like nausea. 


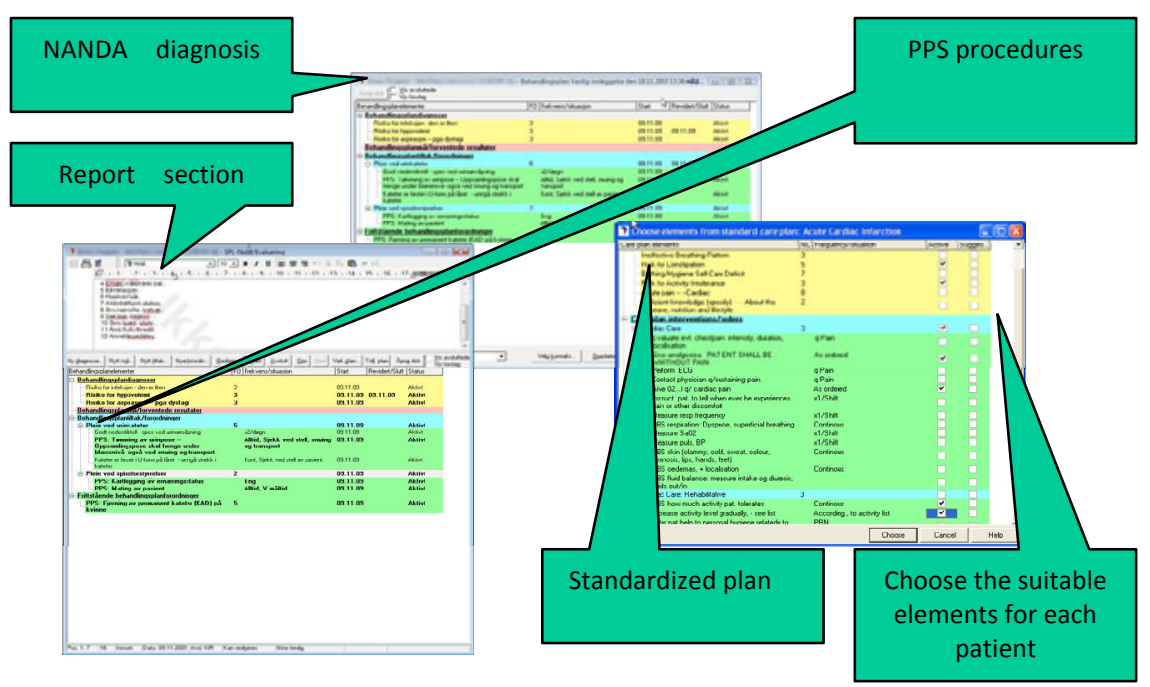

Fig. 2. The nursing plan in different views. The ordinary care plan to the left and the standardized plan to the right.

The well known oral handover was challenged as being ineffective and it was relevant to scrutinize efforts which aimed to replace the oral handover with a written handover, such as a silent handover. "We wished to pursue a silent handover for several reasons. Our goals were to use less time compared to the oral report, hence there would be more time with our patients and improved quality of the documentation" (experienced nurse). Nursing handover has a pivotal role in standardized documentation, both in order to increase efficiency by replacing long, time-consuming oral handovers, and by focusing on the quality of the written documentation. The nurses in Cardiology spoke unanimously in favor of silent handover, as several nurses stated, "We spend much less time on the handovers now than before using oral handovers. We spent too much time on 'small talk.'The socialisation aspect is important, but we always find time to socialize during the shift or during the lunch break."

Consequently, the implementation of the EPR has, with AHUS as with other hospitals, been organized from the vendor's perspective, which has presented the content, the possibilities, and the outcome of using the classification systems. Further, it has been up to local management and user groups to decide which content to apply. At AHUS, several departments grasped and used the standard plans early in the implementation process at the beginning of 2005. The Cardiology department was not the first user, although it was one of the first departments that used several standards systematically alongside using the silent report and, gradually, PPS.

Standard plans were introduced in order to simplify the way problem descriptions should be written. With this as a backbone, the first goal was to increase the number of standardized plans used. Management used a two-step model: first, they focused on the quantity, the number of created standardized plans, without looking at the content, and second, they focussed on quality by scrutinizing the content of the existing plans. Further, 5 to 10 minutes were used each morning to discuss progress and developments. In 
the next phase, they used ten minutes on each of the nurses discussing the content of the plans they had created, focusing on quality. In this way, the users were put centerstage, securing the implementation with the ingenuity of users, and a visible bottom-up approach. A central implication to this is that the effort invested at the time of implementation, the bottom-up approach, has not been continuously repeated given the constant turnover of personnel.

The search engine for the development of standardized plans has received some negative attention for being less than appropriate or accurate, and too timeconsuming. When the nurses were trying to find the appropriate standardized plan, it was obvious that there were not enough search compositions for each standard plan. The right words and numerical orders were needed to find the applicable diagnosis. This procedure was easy when screening for the 17 plans made especially for Cardiology, and also because they consist of the same search words. The problem occurred when they searched for other diagnoses applicable for the patient, but created for another speciality. "If the number of search combinations for each diagnosis were much more numerous, we could find the diagnosis easier by using words that, in using common sense would lead us in the right direction"(inexperienced nurse).

Further, the standardized care plans for cardiology and some of the more generally constructed ones were frequently used by the experienced nurses. In comparison, the standardized plans for other specialities were less frequently used, as "I...always end up with free-text interventions. There is no logical explanation on how to find the diagnosis you are searching for, therefore, I always end up with writing free text" (experienced nurse).

This may not be a big issue when "other specialities" are less relevant to the ward. However, most wards deal with heterogeneous diagnoses where more general standardized plans are developed and definitely should be used. As an example, one of the nurses tried to find a standardized plan for nausea, which is one of the generally developed plans. She searched using different terms such as med-nausea, surgicalnausea, gastric, and nausea without finding the right diagnosis and gave up, went back to the care plan and made nausea a free-text diagnosis. Free-text diagnoses are often used, hence it is better to make free-text plans than no plans at all.

\subsection{The Nurses' Working Routine with the Standardized Care Plan}

Care plans were almost always written by experienced nurses during the first day after hospitalization, usually in the morning. Any updates on other patients were done simultaneously. The experienced nurses' participation in the project period, which included the bottom-up focus of users, has increased their knowledge about the significance and advantages that standardized plans bring to their profession. Hence, they wrote thorough care plans, but the maintenance of already-written plans became insufficient due to the heterogeneity of work practice. If the care plan was used thoroughly on each shift, using free-text, standardized sentences, or both, it is of such a high quality that it could replace all free-text documentation in the EPR. Since the standardized plans also contain PPS procedures, they could give the nurses a complete overview of the patient trajectory. In addition to the improved quality brought forth by using standardized procedures, and standardized sentences, there is a significant efficiency benefit since the plans are very easy to maintain. 
In contrast, the novices that attend the work practice don't get the magnitude of knowledge about standard plans necessary to understand its importance for efficiency and quality of care. Inexperienced nurses typically wrote their reports in the free-text area as 'I write my report mostly using free text in the report section...I haven't learned how to make standardized plans so I try to do updates on the plans written by the more experienced nurses. "In general, a follow-up on this quotation shows that the nurses with less than two years in this particular work practice did not have any education on how or why to use the care plan. The experienced nurses support this, and pinpoint the importance of a new bottom-up initiative in the near future to overcome this problem.

According to the staff of the Cardiology ward, the use of standard plans has decreased after the reallocation process. Hence, general numbers derived from the local IT department covering all clinical departments at AHUS gives a clear indication of the relatively stable use of standardized plans, meaning that the drop was a direct consequence of the reallocation, changes in personnel, and new standards. The longitudinal statistics from a period of six months indicates an overall use close to 20 percent, with slight variations among departments. These numbers fit well with the numbers from the Cardiology department. A cross-section of numbers divided from the Cardiology department indicates that approximately 17 percent of the patients in the ward have standardized plans, measured in the third quarter of 2009. Basing our observation on four consecutive weeks, we see that 40 percent of the patients have a care plan, and that 42 percent of them have a standardized plan.

\subsection{The PPS Integration Process}

The second part of the two-fold project was to integrate a fully operational and automatically updated nursing procedure system: first, with locally adopted procedures and quality documentation, and second, to integrate them with the standardized plans. In contrast to the standardized care plans, the PPS implementation was a top-down decision. The only task performed by local management was to organize the internal education which included two hours of in-house training.

In 2006, AHUS started the PPS project as a decision support system in nursing, and for gaining ISO certification. In addition, the PPS is linked to electronic quality systems (EQS). The PPS database contains 267 detailed procedures for nursing practice, developed by carefully selected professionals around the country, applying to evidence based guidelines for nursing practice.

The existing procedures were evaluated and compared to the PPS procedures with the intention of potentially replacing them with PPS-based procedures. Of the 267 PPS procedures, 191 have replaced existing local ones, 47 have been adjusted to local practices, 26 were not recommended for use at AHUS, and 31 received recommendations for additional procedures. The procedures are further upgraded once a year. Since 2007, PPS has been integrated with the standardized care plan, providing a link between NIC and relevant PPS procedures. The direct integration with the standardized care plan confirmed the hospital's general intention to increase the quality of nursing work with a focus on efficiency. PPS could be accessed on four different levels: from the AHUS intranet, from the main page of the EPR, from the care plan, and from the standardized plans. For entry of a NANDA diagnosis, the standard provides 
the opportunity to choose from a number of predefined interventions appropriate to the patient's condition. Linked to some of the interventions in the standardized plan are short cuts to PPS procedures (see Figure 3). When one specific intervention is selected in the continuous list and the OK button is pressed, the procedure is Webbased with all descriptions attached.

Further, the departments use small handwritten manuals containing summaries of the most frequently used PPS and EQS procedures especially developed for new personnel as a substitute for PPS. Newcomers do not have to chase down the other nurses to access the procedures, and the procedures are followed as prescribed in the PPS. The problem with this method is the possibility of neglecting new or updated procedures.

One problem with the PPS procedures attached to the standardized plans is that there is nothing visual that explains whether a procedure has been updated or not; therefore, the system now demands that the user access PPS occasionally to catch any recent updates. There is also red light telling you to check out any of the attached procedures. All PPS procedures are also integrated in EQS and can be accessed from there.

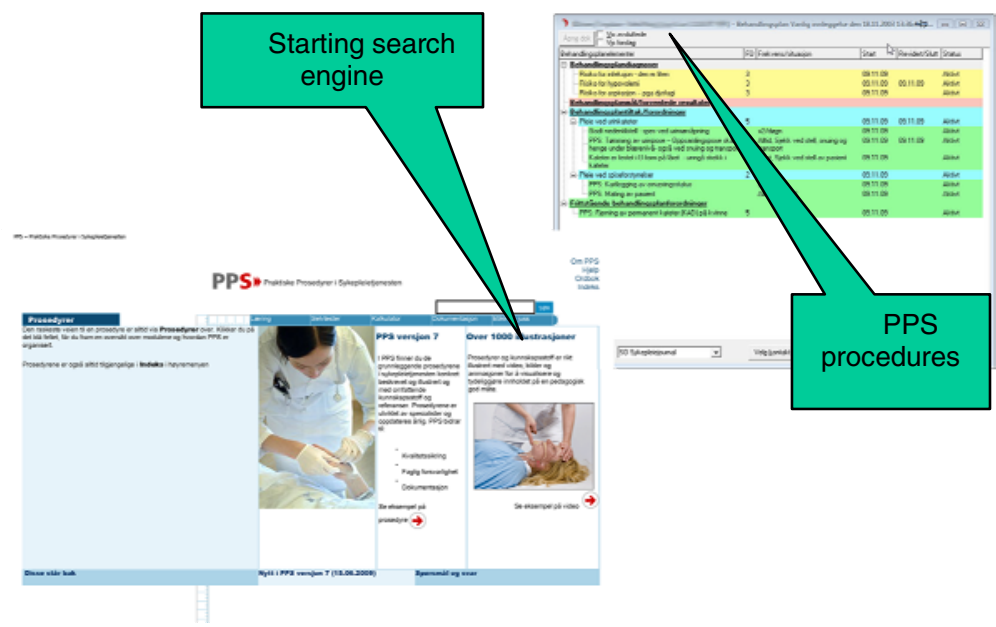

Fig. 3. The PPS front-page with access to the search engine. AHUS employees have access from the intranet and several possibilities in the EPR. This further illustrates standard care plans including direct access to PPS through the selected intervention.

One potential advantage of PPS is that the novices are very skilled in using it. PPS training has been a significant part of their training to become a nurse through the education program. Unfortunately, the experienced nurses and assistants in this department do not use PPS very often. One reason for this is that it is difficult to find the correct procedure, as one nurse explains, "Finding the right procedure in the PPS search engine is not based on intuition. It becomes too time consuming, It is often better to use the handwritten manual or ask the more skilled nurses."

Another challenge is the way the PPS is tightly connected to the standardized plans. The top-down initiative PPS, which has been a part of the education of nurses 
on a national level for several years, and further top-down because of the tight anchorage to hospital management's need for standardized procedures as quality indicators, doesn't fit the use of the bottom-up initiative that exists among the experienced nurses in using standard plans. For instance, given the different level of education and knowledge among the two groups of nurses, and thereby the adoption of the different standards, there will be transversal use. Direct access to PPS through standard plans could have reduced all nurses' difficulties in finding the applicable procedures in the database despite in-house training. The problem is that novices use the care plan mostly as an extension of what the experienced nurses have produced. As one nurse states, "I don't use PPS much in my daily routines, and I don't have the knowledge required to make standardized care plans. I see the opportunities that lie in standardized plans and the direct access to PPS procedures as extremely valuable for us novices. The instructions are very easily accessible." The novices use the PPS procedures linked to the standard plan created by the experts. The experienced nurses used the standardized plans frequently, but they seldom used the associated PPS procedures, for two reasons. First, the procedures were connected to NIC interventions that were frequently used, meaning that the nurses were comfortable with the content of the procedures. Second, when they did not find the correct NANDA diagnosis, they did not find the PPS procedures attached to the predefined interventions.

\section{Discussion and Conclusion}

Perspectives on standardization of work tend to be polarized: either standardization is perceived as a typically top-down effort promoting best practices or, as often argued in socio-technical approaches to ICT in health care (Ellingsen et al. 2007; Timmermans and Berg 1997), standardization is futile, as health work is inherently situated and thus unique.

The perspective emerging from our work provides a theoretically more appealing and empirically more compelling middle position. Standardized work-we focus on nursing practices-is neither straightforward nor uncontroversial. Yet it is not impossible. Essential to standardization with the use of IT in practice, as suggested by our case, is the co-constructing of the standards, in part imposed from the top, in part provided by the active involvement and ingenuity of users.

Throughout our case, we have described scenarios where the focus has been centered on the EPR in use, the IT-based nursing module: two cases, the standard plan, and the PPS.

\subsection{The Co-construction of Standardization}

As claimed, it is vital in IS research to move the focus from the standardization of artefacts and products to include standardized IS-embedded work in the service sector (Ellingsen et al. 2007). Theoretically, we move from the typical top-down perspective to a co-constructive perspective in which standardization and work practice mutually shape and constitute each other. With the introduction of electronic nursing documentation, the information work has shifted from a chronological status note to process-oriented, structured documentation where the EPR care plan, especially the standardized plan, is 
positioned at center stage. First, the reallocation process introduced several new standards. As a result, management wished to invest more effort in the silent handover, finding it crucial to uphold the quality of this part of the documentation process to avoid total collapse in the ongoing work with other standards such as the standard plan. The strategy chosen by the management in the Cardiology ward was built on the established success factors of the silent handover, the accuracy of the documentation, the structure of care plans, and the categorization of standardized plans.

Pursuing the silent handover system was important to enable reliance on written information, since the long-standing oral transformation processes no longer existed. The silent handover is considered a success, and all clinical personnel support its benefits as providing a powerful tool to improve efficiency as well as quality of documentation, which in turn depends on a well-functioning EPR system.

Crucial to the success of this long-term process has been a bottom-up perspective on the implementation of care plans, which has constituted an enhancement of performance among experienced nurses. At the time of integration, the introduction of standard plans was achieved appropriately, with a bottom-up approach that included the ingenuity of the users. Nurses who experienced this period and who have continued working at the ward have become the best ambassadors for future development.

\subsection{The Potential in the Novice-Expert Relationship}

According to the Dreyfus model, nursing practice is shaped and developed by expert nurses, and nurses in general pass through four steps of education before becoming an expert. The five layers of competence are novice, advanced beginner, competent, proficient, and expert (Orlikowski and Baroudi 1991). Hence, in all work practices including hospital departments, there is a differentiation in skills among personnel. For instance, in this case, novices enter the work practice with skill in the use of PPS. We, therefore, find the integrated standard PPS interesting to our case. Up until now, the effect of the PPS integration has failed to materialize, at least in clinical terms and according to the logs, local evaluation of PPS, and interpretations from the user. The top-down perspective utilized in the implementation of PPS has not contributed to the use of procedures, which at this point, and after more than three years, is limited. For PPS to become a success, the interdependency between the PPS procedures integrated with the EPR nursing plans is crucial. We find this interesting, as the novices in the department have skills from which the experts could benefit in the long term depending on the future integration of standard plans with the PPS. The conceptuallization in Benner's (1984) book on the Dreyfus model from novices to experts is interesting with regard to our case in the discussion of further development, expectations for the future of integrated care plans, and PPS. The Dreyfus model does not consider the possibility that the novices could be experts in certain areas such as the use of PPS, and because of recent integration in education. In this way, the expert versus novice relation becomes a bidirectional synergic process, were novices enter the work practice as skilled PPS users, with additional knowledge that the experts could learn from, which again could balance the novice-expert relation. Hopefully, this restructuring will prove fruitful in the future, building on the novices' skills in using IT-based tools for standardization of nursing plans, and the way interdependency gradually makes them become equals. 


\subsection{The Effect of an Array of Coordinated Standardization Efforts}

A new perspective has been the efforts made to overcome heterogeneity in work practice, which seemingly could contribute to the co-construction of standardization (Ellingsen et al. 2007). Exploration of the effects of these standards made it clear that the achieved effect, in relation to increasing both efficiency and quality of work processes, could end up being inherently positive. These standards show the interdependent effect on the IT-based standards installed because of a positive contribution to an easier work practice for nurses. For instance, the generic pool for personnel has secured a continuous flow of substitute nurses who know the environment, routines, and permanent staff. The way these nurses are reused in the departments ensures continuity of care while at the same time allowing the permanent personnel to concentrate on their work process instead of apprenticeship training. Further, the generic wards decrease the number of patients with short-term trajectories of less than 24 hours, which makes the continuity of documentation more transparent. In the long term, these efforts could be a strong contribution to an increased focus on documentation as the nurses' work trajectory becomes more visible. In terms of efficiency, these efforts make work practice more transparent, both for the permanent staff and for others.

The standardization of blood specimen collection has a similar character, even if the decentralization process has failed to compensate for the blood specimen collection. Hence, it has become more transparent and to a different degree organized into the daily routines of the nurses, giving them more time with the patients. Further, the patient does not need to relate so much to continuously alternating personnel. Along this line of standardization, both the transparency and the fixed routines contribute to less work pressure and predictability that positively influence other standards.

The entanglement of different standards, technological and work-process oriented, increases the potential long-term profit when the goal is highly standardized, efficient, and high quality work trajectories, under which conditions the nurses could easily plan and execute the work. The different standards operating in the same working environment contribute to the success of the other standards and underscore the importance of co-constructed standards.

\section{References}

Ash, J.S., Berg, M., Coiera, E.: Some Unintended Consequences of Information Technology in Health Care: The Nature of Patient Care Information System-Related Errors. J. Am. Med. Inform. Assoc. 11, 104-112 (2004)

Benner, P.: From Novice to Expert. Addison-Wesley Publishing Company Inc., Boston (1984)

Berg, M.: Rationalizing Medical Work: Decision Support Techniques and Medical Practices. MIT Press, Cambridge (1997)

Berg, M.: Patient Care Information Systems and Health Care Qork: A Sociotechnical Approach. Int. Journal of Medical Informatics 55, 87-101 (1999)

Bowker, G.C., Star, S.L.: Sorting Things Out. The MIT Press, Cambridge (1999)

Bulechek, G.M., Butcher, H.K., Dochterman, J.M.: NIC: Nursing Interventions Classification, 5th edn. Elsevier, New York (2008)

Button, G., Harper, R.H.R.: Taking the Organization into Accounts. In: Button, G. (ed.) Technology in Working Order. Studies of Work, Interaction, and Technology, pp. 98-107. Routledge, London (1993) 
Ellingsen, G., Monteiro, E., Munkvold, G.: Standardization of Work: Co-constructed Practice. The Information Society 23(5), 309-326 (2007)

Forsythe, D.E.: It's Just a Matter of Common Sense: Ethnography as Invisible Work. Computer Supported Cooperative Work, 127-145 (1999)

Hanseth, O., Lundberg, N.: DesigningWork Oriented Infrastructures. Computer Supported Cooperative Work 10(3/4), 347-372 (2001)

Hanseth, O., Monteiro, E., Hatling, M.: Developing Information Infrastructure Standards: The Tension between Standardization and Flexibility. Science Technology \& Human Values 21(4), 407-426 (1996)

Heps $\varnothing$, V., Monteiro, E.: Ecologies of E-Infrastructure. JAIS 10(5), 430-446 (2009)

Hughes, D.: When Nurse Knows Best: Some Aspects of Nurse/Doctor Interaction in a Casualty Department. Soc. Hea. Illn. 10, 1-22 (1988)

IOM: Crossing the Quality Chasm: A New Health System for the 21st Century. Institute of Medicine. National Academy Press, Washington (2001)

Kalra, D.: Electronic Health Record Standards. IMIA Yearbook of Medical Informatics, 136-144 (2006)

Klein, H., Myers, M.: A Set of Principles for Conducting and Evaluating Interpretive Field Studies in Information Systems. MIS Quarterly 23(1), 67-94 (1999)

Malterud, K.: Kvalitativ metode I medisinsk forskning: 2. utgave. Universitetsforlaget (2003)

NANDA-1: Nursing Diagnosis: Definitions and Classification 2007-2008. NANDA International, Kaukauna (2007)

Orlikowski, W., Baroudi, J.: Studying Information Technology in Organizations: Research Approaches and Assumptions. Information Systems Research 2(1), 1-28 (1991)

Pollock, N., Williams, R., D'Adderio, L.: Global Software and its Provenance: Generification Work in the Production of Organizational Software Packages. STS 37(2), 254-280 (2007)

Schmidt, S.K., Werle, R.: Coordinating Technology. Studies in the International Standardization of. MIT Press, Telecommunication. Cambridge (1998)

Timmermans, S., Berg, M.: The Gold Standard: The Challenge of Evidence-Based Medicine and Standardization in Health Care. Temple University Press, Philadelphia (2003)

Timmermans, S., Berg, M.: Standardization in Action: Achieving Universalism and Localization through Medical Protocols. Social Studies of Science 27, 111-134 (1997)

Ure, J., Proctor, R.: The Development of Data Infrastructure for E-Health: A SocioTechncal Perspective. JAIS 10(5) (2009)

Voutilainen, P., Isola, A., Muurinen, S.: Nursing Documentation in Nursing Homes: State-ofthe-Art and Implications for Quality Improvement. Scand. Journal Caring Sci. 18, 72-81 (2004)

Walsham, G.: Interpretive Case Studies in IS Research: Nature and Method. Eu. J. Inf. Systs. 4, 74-81 (1995)

\section{About the Authors}

Rune Pedersen is a Ph.D. student in the program on E-health and Telemedicine at the University of Tromsoe, Norway. He is in his fourth year of studies and is writing his thesis, which he expects to complete by March 2012. Before starting his Ph.D., he worked as a research associate at the University Hospital of North Norway, educated as a nurse with a Master's degree in Public health. His research interests center on the use of electronic patient records and, in particular, nursing work, IT and work process standards, and IT-based classification systems. He has published in several 
conferences, including the Information Systems Research Seminar in Scandinavia, the International Conference on Information Technology in Health Care, and the European Conference on Information Systems.

Gunnar Ellingsen is a post-doctoral student in the program on E-health and Telemedicine at the University of Tromsoe, Norway. Before completing his Ph.D. in 2003, he worked for several years as an IT consultant at the University Hospital of North Norway. His research interests center on the design and use of electronic patient records in hospitals. He has published articles in Information and Organization, Journal of Computer Supported Cooperative Work, Methods of Information in Medicine, Scandinavian Journal of Information Systems, International Journal of IT Standards \& Standardization Research, and British Medical Journal.

Eric Monteiro is a professor at the Department of Computer and Information Systems at the Norwegian University of Science and Technology. He is broadly interested in organizational transformations and ICT in general, and issues of standardization and globalization in particular. His publication outlets include MIS Quarterly, Journal of CSCW, Science, Technology \& Human Values, Information and Organization, Methods of Information in Medicine, The Information Society, and Scandinavian Journal of Information Systems. 